\title{
Derechos laborales de las mujeres con una visión de género. Caso enfermería
}

\author{
Labor rights of women with a gender vision. Nursing case \\ Direitos trabalhistas das mulheres com uma visão de gênero. \\ Caso de enfermagem
}

\author{
Cecilia Sinche \\ csinche@hotmail.com \\ Recibido septiembre 2018 / Revisión octubre 2018 / Aceptado 1 de enero 2019
}

\begin{abstract}
RESUMEN
Introducción: El acceso de la mujer al trabajo, sigue definido por la división del trabajo en función del género. Es preciso revisar los derechos laborales y sexuales de la mujer trabajadora, caso especial el de la enfermera. Objetivo. Realizar una revisión de la literatura sobre derechos laborales y sexuales de las mujeres con una visión de género, en particular el de la enfermera. Materiales y Método : Revisión de la literatura virtual, en un metabuscador (google) y bases digitales, Biblioteca Virtual de Salud (BVS) y SciELO, Google Scholar y repositorios institucionales de la Flacso, Universidad Andina; de portales del Fondo de Población de las Naciones Unidas (UNFPA), la Organización Internacional del Trabajo (OIT), del Ministerio de Salud Pública y del Trabajo de Ecuador y otras instituciones de interés en temas laborales y de derechos de los trabajadores, mujeres y género. Resultado y Discusión: Las organizaciones gremiales ante la complejidad del trabajo de enfermería, la exposición a riesgos de toda índole en el lugar de trabajo y la necesidad de hacer valer sus derechos, se han ocupado de expresar en un documento, los derechos laborales de los profesionales para ejercer una práctica segura y guardan relación con los derechos humanos en general. Conclusiones: Las profesionales de enfermería están expuestas al denominado techo de cristal, lo cual se evidencia en la dificultad para acceder a cargos directivos en la gestión de salud. El desarrollar una conciencia colectiva dirigida a superar dificultades en el disfrute de los derechos es un paso importante para una enfermería autónoma.
\end{abstract}

Palabras clave: enfermería; jurisprudencia; identidad de género

\begin{abstract}
Introduction: Women's access to work is still defined by the division of labor according to gender. It is necessary to review the labor and sexual rights of working women, especially that of the nurse. Objective. Conduct a review of the literature on labor and sexual rights of women with a gender vision, particularly that of the nurse. Materials and Method. Review of the virtual literature, in a metasearch engine (google) and digital bases, Virtual Health Library (VHL) and SciELO, Google Scholar and institutional repositories of Flacso, Andean University; of portals of the United Nations Population Fund (UNFPA), the International Labor Organization (ILO), the Ministry of Public Health and Labor of Ecuador and other institutions of interest in labor issues and rights of workers, women and gender. Result and Discussion. Trade union organizations in view of the complexity of nursing work, exposure to risks of all kinds in the workplace and the need to enforce their rights, have expressed themselves in a document, the labor rights of professionals to practice a safe practice and are related to human rights in general. Conclusions. Nursing professionals are exposed to the so-called glass ceiling, which is evidenced by the difficulty in accessing managerial positions in health management. Developing a collective conscience aimed at overcoming difficulties in the enjoyment of rights is an important step for autonomous nursing.
\end{abstract}

Key words: nursing; jurisprudence; gender identity

CS: Universidad de Cuenca, Ecuador 


\section{RESUMO}

CS: Universidad de Cuenca, Ecuador

\begin{abstract}
Introdução: O acesso das mulheres ao trabalho ainda é definido pela divisão do trabalho de acordo com o gênero. É necessário revisar os direitos trabalhistas e sexuais das mulheres trabalhadoras, especialmente as da enfermeira. Objetivo. Realizar uma revisão da literatura sobre direitos trabalhistas e sexuais de mulheres com uma visão de gênero, particularmente a da enfermeira. Materiais e Métodos: Revisão da literatura virtual, em um mecanismo de meta-pesquisa (google) e bases digitais, Biblioteca Virtual em Saúde (BVS) e SciELO, Google Scholar e repositórios institucionais de Flacso, Universidade Andina; portais do Fundo das Nações Unidas para a População (UNFPA), a Organização Internacional do Trabalho (OIT), o Ministério da Saúde Pública e Trabalho do Equador e outras instituições de interesse em questões trabalhistas e direitos dos trabalhadores, mulheres e sexo. Resultado e discussão. As organizações sindicais, considerando a complexidade do trabalho de enfermagem, a exposição a todos os tipos de riscos no local de trabalho e a necessidade de fazer valer seus direitos, expressaram-se em um documento, os direitos trabalhistas da profissionais a praticar uma prática segura e estão relacionados aos direitos humanos em geral. Conclusões. Os profissionais de enfermagem estão expostos ao chamado teto de vidro, o que é evidenciado pela dificuldade de acesso às posições gerenciais na gestão em saúde. Desenvolver uma consciência coletiva voltada para a superação de dificuldades no gozo de direitos é um passo importante para a enfermagem autônoma.
\end{abstract}

Palavras-chave: enfermagem; jurisprudência; identidade de gênero

\section{INTRODUCCIÓN}

$\mathrm{E}$ l acceso de la mujer al trabajo, sigue definido por la división del trabajo en función del género. División entre trabajo reproductivo o doméstico, no retribuido ni valorado, asignado habitualmente a las mujeres y el trabajo productivo o empleo, retribuido y valorado socialmente, asignado tradicionalmente a los hombres (1).

Las actividades reproductoras que realizan las mujeres no son remuneradas, en muchos países no están cubiertas por la legislación laboral, como consecuencia, el trabajo realizado por las mujeres queda excluido de las estadísticas de empleo y de rentas (2). Es preciso revisar las tareas desde una perspectiva de género, de forma que las funciones productivas $y$ reproductoras en el hogar, la comunidad o el lugar de trabajo puedan ser compartidas equitativamente porhombres y mujeres. Como también, conocer el Derecho Laboral y con ello los derechos humanos en particular, los derechos laborales $y$ sexuales de la mujer trabajadora, específicamente el de la enfermera.

La enfermería, es una profesión mayoritariamente femenina, donde la enfermera se enfrenta a los retos de un trabajo exigente, que ocasiona riesgos a su salud y además, se enfrenta a las múltiples actividades asignadas a su género.

Se hace necesario estudiar las implicaciones para las profesionales de enfermería en su condición de mujer trabajadora, pero también, como responsable en el trabajo invisible en el hogar, que además se suma a la carga biológica que implica la reproducción biológica y los procesos menstruales.

El objetivo fue realizar una revisión de la literatura sobre derechos laborales y sexuales de las mujeres con una visión de género, en particular de la enfermera.

\section{MATERIALES Y MÈTODOS}

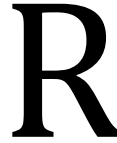

evisión de la literatura virtual, en un metabuscador (google) y bases digitales, Biblioteca Virtual de Salud (BVS) y SciELO, Google Scholar y 
repositorios institucionales de la Flacso, Universidad Andina; de portales del Fondo de Población de las Naciones Unidas (UNFPA), la Organización Internacional del Trabajo (OIT), del Ministerio de Salud Pública y del Trabajo de Ecuador y otras instituciones de interés en temas laborales y de derechos de los trabajadores, mujeres y género. Se revisaron, en los idiomas inglés, portugués y español según descriptores de derechos laborales (labor rights), género (gender) and mujer (woman).

\section{DISCUSIÓN}

\section{Antecedentes del derecho laboral}

El Derecho Laboral surgió a consecuencia de la necesidad de regular las relaciones entre los trabajadores y el empleador, con el objeto de establecer los derechos y obligaciones tanto uno para el otro, sostener el equilibrio social y evitar las condiciones que vulneraban los derechos de los trabajadores. Es un derecho protector de la clase trabajadora, que norma las fuentes de empleo para que permitan un nivel de vida digno al trabajador y su familia (1).

Un antecedente en Derecho Laboral fue la Revolución Industrial, que representó para el mundo el paso del trabajo artesano al obrero, debido al descubrimiento e invención de nuevas tecnologías y máquinas, que, si bien aumentaban la productividad, para el trabajador, significó el cumplimiento de largas jornadas laborales que sobrepasaban las dieciséis horas diarias así como la explotación de la mano de obra femenina e infantil, que se expresaba en una menor remuneración con respecto al hombre (2).

En general, durante la época de la Revolución Industrial, las condiciones de trabajo se caracterizaban por: extenuantes jornadas, en difíciles condiciones con falta de higiene y seguridad, que ocasionaron un aumento de los accidentes de trabajo y enfermedades ocupacionales, que dieron lugar a las protestas de los obreros e impulsó el movimiento sindical en busca de mejores condiciones laborales. $\mathrm{Al}$ terminar la Primera Guerra Mundial, se creó la Organización Internacional del Trabajo (OIT) en abril de 1919, organización adscrita a las Naciones Unidas que se ocupa de los asuntos concernientes al trabajo y las relaciones laborales (1).

Antes de la fundación de la OIT, en 1919, varios países latinoamericanos habían dado muestras de su interés en la legislación laboral, al adoptar la jornada de ocho horas diarias de trabajo y 48 semanales, entre ellos: Panamá, en 1914 el primer país de la región que reconoció el régimen de las ocho horas de trabajo; Uruguay, en 1915; Ecuador, en 1916; México con el artículo 123 de la Constitución de 1917 y Perú, en enero de 1919 (3).

Al finalizar la Segunda Guerra Mundial, los horrores vividos y los juicios de Núremberg y Tokio, mostraban la necesidad de regular el concepto de derechos humanos $y$ en especial, establecer cuáles eran. Por ello en 1945, se creó la Organización de las Naciones Unidas (ONU), instancia facultada para tomar medidas sobre los problemas que enfrentaba la humanidad (3).

Uno de los primeros trabajos de las Naciones Unidas fue la elaboración de la Declaración Universal de los Derechos Humanos, aprobada el 10 de diciembre de 1948, un texto cuya redacción estuvo sujeta a muchas discusiones por parte de la representación de cada país miembro de la organización, ya que las visiones eran diferentes acerca de sus contenidos (4). Una serie de tratados internacionales y otros instrumentos adoptados desde 1945, han extendido el derecho internacional de 
los derechos humanos. Entre los cuales figuran: la Convención para la Prevención y la Sanción del Delito de Genocidio (1948), la Convención Internacional sobre la eliminación de todas las formas de Discriminación Racial (1965), la Convención sobre la eliminación de todas las formas de Discriminación contra la Mujer (1979), la Convención sobre los Derechos del Niño (1989) y la Convención sobre los Derechos de las Personas con Discapacidad (2006) (5).

Según la ONU, "Los derechos humanos son inherentes a todos los seres humanos, sin distinción alguna de raza, sexo, nacionalidad, origen étnico, lengua, religión o cualquier otra condición" (4).

En 1998, la OIT emitió una Declaración sobre los Principios y Derechos Fundamentales en el Trabajo (6), en la que estableció la obligatoriedad que tienen los miembros de esta organización a respetar y cumplir con los principios relativos a los derechos fundamentales que son objeto de los convenios de protección al trabajo, es decir: a) La libertad de asociación y la libertad sindical, y negociación colectiva. b) La eliminación de todas las formas de trabajo forzoso $\mathrm{u}$ obligatorio. c) La abolición efectiva del trabajo infantil. d) La eliminación de la discriminación en materia de empleo y ocupación.

En América Latina, el compromiso de eliminación de la discriminación en el empleo, se sumó a un conjunto de medidas para erradicar la discriminación contra las mujeres en todas sus formas.

\section{Derechos de las mujeres}

Históricamente, las mujeres estuvieron excluidas de la educación y destinadas a la reproducción de las relaciones de poder en el hogar. Su función era el aprendizaje de las tareas domésticas, alejadas del proceso de desarrollo de la sociedad, no se aceptaban féminas en las primeras universidades que datan del siglo y fue sólo en el siglo XX, que se aceptó su ingreso (7).

Para Zamudio (8), fue en el escenario educativo donde se evidenció con más fuerza la reproducción de las diferencias de género, por ser considerado espacio exclusivo para la formación profesional de varones. En este sentido, el Programa de las Naciones Unidas para el Desarrollo Sostenible (PNUD), promulgó como quinto objetivo del milenio "la igualdad de género", con el fin de erradicar todas las formas de discriminación contra las mujeres y niñas.

La Organización de las Naciones Unidas (ONU), desde la adopción de su carta fundacional ha apoyado el desarrollo de los derechos de las mujeres, en la dignidad y el valor de la persona humana sin distinción de sexo, raza, idioma y religión $(9,10)$. (Tabla 1$)$. 
Tabla 1. Avances de la ONU en la protección de los derechos de la mujer

\begin{tabular}{llll}
\hline Año & Plataforma & Objetivos/logros \\
\hline 1946 & $\begin{array}{l}\text { Comisión de la condición Jurídica y } \\
\text { Social de la Mujer. }\end{array}$ & $\begin{array}{l}\text { Desarrollar las políticas mundiales dedicadas a la } \\
\text { igualdad del género. }\end{array}$ \\
\hline 1952 & $\begin{array}{l}\text { Convención sobre los Derechos } \\
\text { Políticos de la Mujer. }\end{array}$ & $\begin{array}{l}\text { Derecho a votar, derecho a ser elegida y } \\
\text { desempeñar cargos públicos. }\end{array}$ \\
\hline 1975 & $\begin{array}{l}\text { Año Internacional la Mujer, 1era } \\
\text { Conferencia Mundial sobre la Mujer. }\end{array}$ & $\begin{array}{l}\text { Década de la mujer y un fondo voluntario para la } \\
\text { década: 1976-1985. }\end{array}$ \\
\hline 1979 & $\begin{array}{l}\text { Convención sobre la eliminación de } \\
\text { todas las formas de discriminación } \\
\text { contra la mujer. }\end{array}$ & $\begin{array}{l}\text { La cultura y tradiciones como factores influyentes } \\
\text { en la construcción de los roles de género, reafirma } \\
\text { los derechos de reproducción de la mujer. }\end{array}$ \\
\hline 1980 & $\begin{array}{l}\text { Segunda Conferencia Mundial sobre } \\
\text { la Mujer. }\end{array}$ & $\begin{array}{l}\text { Medidas para asegurar los derechos de la mujer; } \\
\text { la herencia, custodia de los hijos y pérdida de la } \\
\text { nacionalidad. }\end{array}$ \\
\hline 1985 & $\begin{array}{l}\text { Conferencia Mundial para el examen } \\
\text { y la evaluación de los logros del } \\
\text { decenio de la ONU para la mujer. }\end{array}$ & $\begin{array}{l}\text { Revisión y evaluación de los logros alcanzados } \\
\text { por la década de mujer de las Naciones Unidas: } \\
\text { igualdad, desarrollo y paz. }\end{array}$ \\
\hline 1993 & $\begin{array}{l}\text { Declaración sobre eliminación de la } \\
\text { violencia contra la mujer. }\end{array}$ & $\begin{array}{l}\text { Define violencia contra la mujer, reafirma los } \\
\text { derechos para evitar violencia de la mujer. }\end{array}$ \\
\hline 1995 & $\begin{array}{l}\text { Cuarta Conferencia Mundial sobre la } \\
\text { mujer. }\end{array}$ & $\begin{array}{l}\text { Derechos de la mujer como derechos humanos, } \\
\text { acciones sobre mujer y pobreza, educación, salud, } \\
\text { violencia, la mujer en los conflictos armados, } \\
\text { economía, propiedad y poderes de decisión. }\end{array}$ \\
& $\begin{array}{l}\text { Promover la igualdad de género y el } \\
\text { empoderamiento de la mujer. }\end{array}$ \\
\hline 2000 & $\begin{array}{l}\text { La Declaración del Milenio Naciones } \\
\text { Unidad } \\
\text { a/RES/552. }\end{array}$ & $\begin{array}{l}\text { Entidad de la ONU para la igualdad de género y el } \\
\text { empoderamiento de la mujer. }\end{array}$ \\
\hline Creación ONU mujeres. & Resolución
\end{tabular}

La conciencia social que se desarrolló sobre los derechos de la mujer, en especial la importancia de la maternidad y cuidado del recién nacido, llevó a la OIT a adoptar diferentes convenios (tabla 2), orientados a la igualdad de remuneración, a la no discriminación en el empleo y la ocupación, a la protección para cumplir con las responsabilidades familiares, protección de la maternidad y cuidados de los miembros de la familia (9).

Tabla 2. Convenios OIT para los derechos de las mujeres trabajadoras

\begin{tabular}{|c|c|c|}
\hline $\mathbf{N}^{\circ}$ & Convenio & Derecho \\
\hline 100 & Igualdad de remuneración. & $\begin{array}{l}\text { Las mujeres tienen derecho a percibir el mismo } \\
\text { salario que los hombres cuando realizan un } \\
\text { trabajo del mismo valor que éstos. }\end{array}$ \\
\hline 111 & $\begin{array}{l}\text { Discriminación en el empleo y } \\
\text { ocupación. }\end{array}$ & $\begin{array}{l}\text { Ninguna persona puede ser discriminada en su } \\
\text { empleo u ocupación por motivos de raza, color, } \\
\text { sexo, ideas políticas, creencias religiosas, } \\
\text { condición social. }\end{array}$ \\
\hline 156 & $\begin{array}{l}\text { Trabajadores con responsabilidades } \\
\text { familiares. }\end{array}$ & $\begin{array}{l}\text { Todas las trabajadoras y trabajadores con } \\
\text { responsabilidades familiares, tienen derecho a: } \\
\text { protección especial y a no ser discriminados en el } \\
\text { empleo y ocupación por esta condición. }\end{array}$ \\
\hline 183 & Protección de la maternidad. & $\begin{array}{l}\text { Protección de la maternidad, con el objetivo de } \\
\text { seguir promoviendo, la igualdad de todas las } \\
\text { mujeres en el trabajo, la salud y la seguridad de la } \\
\text { madre y el niño. }\end{array}$ \\
\hline
\end{tabular}


Una mayor especificación de las ramas, en las que se encuentran un mayor número de mujeres son: otros servicios (servicio doméstico, servicios sociales y de salud, enseñanza), comercio, manufactura y servicios financieros. Mientras que las ramas mayoritariamente masculinas, son: la construcción, la industria, la minería, la rama agropecuaria y las de electricidad y transporte. Los hombres tienen una mayor diversificación laboral (8).

La composición habitual de algunos sectores productivos, da cuenta de la segmentación del mercado laboral, de acuerdo a la que se perpetúa el patrón de género, tanto por la cantidad de mujeres que concentra, como en el menor valor económico y de reconocimiento que se le confiere a su trabajo frente a las ramas proverbialmente masculinas (11). La mujer ha demostrado estar en capacidad de asumir retos en cualquier sector productivo de la sociedad, de hecho, de contribuir al desarrollo social, pero para consolidar esta participación debe recibir apoyo.

\section{Los derechos laborales y sexuales de las mujeres en Ecuador}

Los derechos laborales están contemplados en la Constitución de la República del Ecuador, y en su artículo 33 determina que el trabajo es un derecho, un deber social y un derecho económico, fuente de realización personal y base de la economía, donde el Estado garantizará a las personas trabajadoras, el pleno respeto a su dignidad, una vida decorosa, remuneraciones y retribuciones justas y el desempeño de un trabajo saludable y libremente escogido o aceptado $(11,12)$. Los derechos laborales consagrados en el ordenamiento jurídico de cada país, como garantías individuales y sociales de la persona trabajadora, sin ninguna condición, tienen un fundamento común que es el derecho al trabajo.

\section{Las trabajadoras del Ecuador son amparadas por leyes como:}

1. La LOSEP (Ley Orgánica del Servicio Público) que tiene por objetivo, fomentar el desarrollo profesional, técnico y personal de las y los servidores públicos, sustentado en la igualdad de derechos, oportunidades y la no discriminación (13).

2. El Código del Trabajo, texto fundamental desde el 2005. Para finales del año 2012, se incluyeron varios artículos relevantes para la mujer (14) Licencias por maternidad $\mathrm{y}$ por paternidad, jornada reducida para las madres lactantes, protección de la mujer embarazada.

3. La Ley Orgánica para la Justicia Laboral y Reconocimiento del Trabajo en el Hogar ("LOJLRTH") aprobada en 2015 y considerada como un hito histórico, por su alcance en lo relativo a derechos de las embarazadas y de las personas que realizan trabajo doméstico (14).

\section{Los derechos sexuales}

En Ecuador, la integración de las mujeres al mercado de trabajo, no se ha visto acompañada de una redistribución de la carga de trabajo doméstico, de forma equilibrada y no sexista, ellas siguen realizando más trabajo que los hombres, además de cumplir labores a las que se le confiere poca relevancia, secundarias y de menor valor económico, sobre todo el trabajo doméstico y el comercio informal (8).

Los esfuerzos en Ecuador por legislar de manera equitativa en términos de género, con igualdad entre hombres $\mathrm{y}$ mujeres, y equilibrar dichas relaciones, son evidentes en los últimos años (7). La inserción de la mujer al mundo del trabajo, se hace bajo el prisma de la cultura católica, según los preceptos de la religión 
predominante en el país, que contribuye a marcar los roles que deben tener las mujeres, así como también los hombres (9). Las mujeres con su participación en lugares que tradicionalmente le habían sido negados, se hacen visibles como ser político y sujeto social.

\section{La enfermera como trabajadora en el contexto de derechos laborales y sexuales}

La enfermería al ser una profesión predominantemente femenina, tiene una fuerte carga vinculada al género y a la equidad de género. La relación interprofesional se caracteriza por actos sutiles o no, de poder interprofesional, derivados de dos dimensiones: el género (femenino) y el (bajo) estatus y prestigio que guarda en el concierto social, profesional y laboral (15).

Se ha evidenciado que un factor laboral que afecta el trabajo de la enfermera, es el modelo hegemónico imperante, un modelo de atención hospitalaria, donde cobra prevalencia la figura del médico como figura central. Esto no solamente ha propiciado la violencia intragénero, sino también, ha dificultado que el personal de enfermería desarrolle una autonomía profesional, de manera que su liderazgo queda relegado e impacta su reconocimiento profesional y social (15).

La enfermería a través del tiempo, ha sido ejercida fundamentalmente por mujeres, se ha visto determinada por los roles asignados a hombres y mujeres en una sociedad que ha sido construida $y$ liderada desde un esquema patriarcal, que se destaca con fuerza en el ámbito asistencial (16).

\section{Derechos laborales de las enfermeras y enfermeros}

La enfermería ha estado caracterizada por el difícil acceso a los altos cargos directivos en la gestión de salud, a pesar de las competencias profesionales exhibidas, pareciera que es territorio masculino (16). Los estereotipos de género juegan un papel importante; aún se conserva la imagen de la enfermera sumisa, emocional, débil, sumisa, dependiente, sensible, comprensiva o poco competitiva (17). Imagen distorsionada de la actual enfermera empoderada, autónoma $\mathrm{y}$ calificada que presta cuidado, gerencia, investiga, enseña $y$ asume el rol protagónico en la defensa de sus derechos, esa es la enfermera del siglo XXI.

Las organizaciones gremiales ante la complejidad del trabajo de enfermería, la exposición a riesgos de toda índole en el lugar de trabajo y la necesidad de hacer valer sus derechos, se han ocupado de expresar en un documento, los derechos laborales de los profesionales para ejercer una práctica segura y guardan relación con los derechos humanos en general (18).

Son derechos del profesional de enfermería, ejercer su trabajo en libertad, sin discriminación en el grupo profesional. Desempeñar sus intervenciones, en un entorno que garantice la seguridad e integridad personal y profesional. Contar con los recursos necesarios que le permitan el óptimo desempeño de sus funciones. El asumir el cuidado de otros, es una gran responsabilidad con la sociedad que involucra, además, el cuidado de sí mismo y la lucha por los derechos que asisten al profesional de enfermería.

\section{CONCLUSIONES}

$\mathrm{L}$ os derechos laborales y sexuales de la mujer, han evolucionado de forma sostenida en el tiempo, mediante el trabajo conjunto de diferentes organizaciones internacionales y el trabajo de agrupaciones defensoras de los derechos de las mujeres. Ademas; las profesionales de enfermería, están expuestas al denominado techo de cristal, 
lo cual se evidencia, en la dificultad para acceder a cargos directivos en la gestión de salud. Por ello, es que la profesión de enfermería está compuesta en su mayoría por mujeres, con lo cual, hay una fuerte carga vinculada al género y a la equidad de género. Es por eso que debe empoderar a las enfermeras en la defensa de sus derechos laborales y sexuales, es necesario conocerlos, exigirlos y que las instituciones empleadoras cumplan con la obligación legal y moral de respetarlos.

- Conflicto de intereses: Ninguno declarado por la autora.

- Fuente de financiamiento: Autofinanciado.

- Agradecimientos: Ninguno declarado por la autora.

\section{REFERENCIAS BIBLIOGRÁFICAS}

1. Reyes L. Derecho laboral. México: Red Tercer Milenio; 2012. [Consulta 2018 29 de mayo]. Disponible en: https://www.upg.mx/wpcontent/uploads/2015/10/LIBRO-24Derecho_laboral.pdf

2. Ricardo M. Derecho laboral, un enfoque práctico. México: McGrawHill; 2009.

3. Villasmil H. Pasado y presente del derecho laboral latinoamericano y las vicisitudes de la relación de trabajo. Rev. latinoam. derecho soc. 2016. [Consultado 20181 de julio]; 22. Disponible en: <http://www.scielo.org.mx/scielo.ph p?script=sci_arttext\&pid=S187046702016000100008\&lng=es\&nrm=i so>. ISSN 1870-4670

4. Amnistía Internacional. Catalunya. Historia de los Derechos Humanos. [Internet] (Consultado 200828 de mayo]. Disponible en: http://www.amnistiacatalunya.org/e $\mathrm{du} / \mathrm{es} /$ historia/inf-resumen.html
5. Castro J. Discriminación en las relaciones laborales. Algunos casos particulares. Boletín Dirección del Trabajo de Chile. [Internet].2001. [Consultado 201802 de julio]; 146. Disponible en: file://C:/Users/maribel/AppData/Lo cal/Temp/discriminación\%20en\%201 as\%20relaciones\%20laborales-1.pdf

6. Organización de las Naciones Unidas. Derechos Humanos. Serie Tener derechos no basta, №. 8. Venezuela; 2014. [Consultado 201802 de julio] Disponible en: http://www.un.org/es/sections/issue s-depth/human-rights/index.html

7. Gómez D. Principios y derechos fundamentales de los trabajadores en Colombia, inclusión en tratados de libre comercio y breve comparación con los de otros países iberoamericanos. Rev. IUS. Puebla. 2014. [Consultado 201828 de julio); 8(33): 98-160. Disponible en <http://www.scielo.org.mx/scielo.ph p?script=sci_arttext\&pid $=\mathrm{S} 1870$

8. Zamudio A. El acceso de las mujeres a la educación superior. La presencia femenina en la Universidad de Los Andes. Procesos Históricos. Rev de Historia y Ciencias Sociales. 2016; 29: 77-101. Disponible en: http://www.saber.ula.ve/bitstream/h andle/123456789/41589/art4.pdf;jse ssionid=689DBBEE317B764DAAC58B 736BF5C50F?sequence $=1$

9. Organización Internacional del Trabajo. Declaración relativa a los principios y derechos fundamentales en el trabajo y su seguimiento. Conferencia Internacional del Trabajo, 86a. Reunión, 18 de junio de 1998. [Consultado 201827 de agosto]. Disponible en: http://www.ilo.org/declaration/thed eclaration/textdeclaration/langes/index.htm 
10. Valenzuela M. Género, pobreza, empleo y economía informal en Ecuador. [Internet]). Perú: Oficina Regional de la OIT para América Latina y el Caribe; 2006. [Consultado 201827 de agosto]. Disponible en: http://www.worldcat.org/title/emplo yment-challenge-in-latin-americaand-thecaribbean/oclc/714877822\&referer= brief_results

11. Mijares A. Derechos sexuales y reproductivos de las mujeres en América Latina. Pensamiento Iberoamericano. [Internet].2011 [Consultado 201802 de julio]; 9(11):281-291.Disponible en: https://dialnet.unirioja.es/servlet/art iculo? codigo $=3710942$

12. Constitución de la República del Ecuador 2008. [Internet]. Ecuador Legal Online. 2017 [Consultado 2018 27 de julio]. Disponible en: http://www.ecuadorlegalonline.com/ biblioteca/constitucion-ecuador-2008

13. Ley Orgánica del Servicio Público. Registro Oficial Suplemento 294. [Internet].2010 [Consultado 201827 de julio] Disponible en: http://www.oas.org/juridico/pdfs/m esicic4_ecu_org10.pdf

14. Andrés L. Las mujeres y el derecho laboral ecuatoriano desde el enfoque de género. Una mirada a la Ley Orgánica para la Justicia Laboral y el reconocimiento del trabajo del hogar. Revista de Estudios Jurídicos.
[Internet].2018 [Consultado 201826 de agosto]; 4:18-31. Disponible en: http://derecho.udla.edu.ec/calamo/i mages/revistaspdf/Calamo4/2dossierLydiaAndrs.pdf

15. Cárdenas L, Velasco C, Fabela C. Desempeño laboral del personal de enfermería: una visión de género. Rev. Horizontes. [Internet].2012. [Consultado 201804 de agosto]; 14:9$22 . \quad$ Disponible en: http://web.uaemex.mx/revistahorizo ntes/docs/revistas/Vol4/desempeno_ laboral_del_personal.pdf

16. Gallego J. Discriminación de género en enfermería. Universidad de Salamanca. Repositorio Institucional.2009. Disponible en: ttps://gredos.usal.es/jspui/bitstream /10366/80253/1/TFM_EstudiosInter disciplinaresGenero_GallegoGarcia_J.p $\mathrm{df}$

17. Mosqueda A, Paravic T, Valenzuela S. División sexual del trabajo $y$ Enfermería. Index Enferm [Internet].2013 [Consultado 201810 julio]; 22(1-2): 70-74. doi.org/10.4321/S113212962013000100015.

18. Sociedad Española de Enfermería de Urgencia. Derechos de las enfermeras y enfermeros. [Consulta 201802 de julio]. Disponible en: http://www.enfermeriadeurgencias.c om/images/archivos/derechos_enfer meros.pdf 